\title{
Error detection and correction patterns in dementia: A breakdown of error monitoring processes and their neuropsychological correlates
}

\author{
BRIANNE MAGOUIRK BETTCHER, ${ }^{1}$ TANIA GIOVANNETTI, ${ }^{1}$ LAURA MACMULLEN, ${ }^{1}$ AND \\ DAVID J. LIBON ${ }^{2}$ \\ ${ }^{1}$ Psychology Department, Temple University, Philadelphia, Pennsylvania \\ ${ }^{2}$ New Jersey Institute for Successful Aging, University of Medicine and Dentistry New Jersey-School of Osteopathic Medicine, \\ Stratford, New Jersey
}

(Received March 23, 2007; Final Revision September 7, 2007; Accepted September 7, 2007)

\begin{abstract}
Error monitoring is critical to an individual's ability to function autonomously. This study characterized error detection and correction behaviors within the service of everyday tasks in individuals with dementia. Also, the impact of neuropsychological functioning on error detection and correction was examined. Fifty-three participants diagnosed with Alzheimer's disease (AD) or vascular dementia (VaD) were administered a neuropsychological protocol and the Naturalistic Action Test, which requires performance of three everyday tasks. Error detection, correction, and the point at which correction occurred (i.e., microslip-before the error was completed, immediate - just after the error was made, delayed—after performing other task steps) was coded. Dementia participants detected $32.7 \%$ of their errors and corrected $75.8 \%$ of detected errors. Participants were more likely to engage in microslips than delayed corrections. Tests of executive control and language predicted detection and correction variables; moreover, detection and correction were each related to different aspects of executive functioning. Microslips were related to naming ability. AD and VaD patients did not differ on detection/correction variables, and regression analyses indicated that dementia severity and memory abilities were unrelated to detection/correction. The results specify the error monitoring deficits in $\mathrm{AD}$ and $\mathrm{VaD}$ and have implications for improving functional abilities in dementia. (JINS, 2008, 14, 199-208.)
\end{abstract}

Keywords: Awareness, Everyday action, Naturalistic action, Activities of daily living (ADL), Instrumental ADL, Alzheimer's disease, Vascular dementia

\section{INTRODUCTION}

The production of error-laden behavior represents a natural, albeit costly component of everyday human activity. For most people, errors of action simply result in personal hassle and inconvenience; however, for people with dementia, action errors may render devastating consequences that jeopardize autonomy and safety (Rabbit, 1978; Reason, 1990). The ubiquitous and arguably inevitable nature of human error has resulted in a gradual change of focus in the everyday action and rehabilitation literatures. Researchers have acknowledged that eliminating errors may be unrealistic; improving

Correspondence and reprint requests to: Tania Giovannetti, Ph.D., Psychology Department, Temple University, Weiss Hall, 1701 N. $13^{\text {th }}$ Street, Philadelphia, Pennsylvania 19122. E-mail: tgio@temple.edu error detection and correction may offer a more tenable strategy for facilitating everyday functioning in individuals with cognitive impairment (Blavier et al., 2005; Dehaene et al., 1994; Manly et al., 2002). The purpose of this study is to characterize everyday error detection and correction processes in dementia patients. The neuropsychological processes associated with error detection and correction were also examined to elucidate the nature of monitoring deficits and inform rehabilitation techniques.

According to Zapf and Reason (Zapf \& Reason, 1994), the "error handling" process consists of two phases: error diagnosis (i.e., detection) and error recovery (i.e., correction). Recent neuroimaging studies have shown support for this distinction. Error detection has been linked to activity in the anterior cingulate cortex (i.e., "conflict monitoring"), whereas correction has been associated with the lateral pre- 
frontal cortex and/or the basal ganglia (Botvinick et al., 2004; Gehring \& Knight, 2000).

Reason (1990) has described the role of auto-control processes and the environment in error detection. Auto-control processes are automatic physiological indicators that trigger error detection without conscious awareness or control. They occur in the earliest stages of error execution and lead to immediate correction in the majority of cases $(90 \%)$ by healthy individuals (Blavier et al., 2005). Studies have shown that error detection is not disrupted or slowed by the diversion of attentional resources away from the task at hand in healthy controls (Giovannetti et al., 2007; Oomen \& Postma, 2002; Postma, 2000); thus, there is evidence for automatic detection processes. Error detection also may be elicited by environmental restriction or feedback. In these cases, detection usually occurs later, after the error has been fully executed.

The second stage of the "error handling" process involves containing or rectifying the error. In some cases, correction occurs very rapidly, even before an error is completely executed. This form of correction may be triggered by autocontrol detection processes (described earlier). Various terms in the literature are used to refer to this rapid error detection/ correction (e.g., microslip, error sketch; covert repair) (Blavier et al., 2005; Hartsuiker \& Kolk, 2001; Postma \& Kolk, 1993). A real world example of a microslip is stopping short of adding sugar, instead of coffee grinds, to a coffee maker in a cluttered kitchen. The timing of the correction (before the error is fully executed) defines a microslip. A second form of correction occurs later, following the execution of an error. This type of correction may involve more effortful executive functions and problem-solving skills. For example, after mistakenly adding the sugar in the coffee maker, one must generate solutions to remedy or bypass the problem (e.g., remove the sugar, wash the machine, buy a cup of coffee on the way into work, etc.).

Careful analysis of error detection/correction in everyday tasks may help to elucidate error monitoring deficits in neuropsychological patients. For example, a recent study of $\mathrm{JC}$, who demonstrated alien hand symptoms of the right hand following a stroke affecting the left medial frontal lobe and corpus callosum, showed that he did not differ from controls in the proportion of action errors that were corrected with the right (alien) hand (Biran et al., 2006). However, unlike controls, a significant proportion of JC's right-hand errors were detected later, after the error had been fully executed. Thus, JC was capable of rapidly detecting his right hand errors, but he was unable to exert control over his right hand to immediately correct these errors. While preliminary, these findings illustrate how careful characterization of error detection/correction time frames may promote understanding of monitoring deficits (see also Hart et al., 1998 for a detailed analysis of error detection/ correction after traumatic brain injury).

To our knowledge, there is only one published study of everyday error detection and correction in dementia (Giovannetti et al., 2002a). The study showed that dementia participants were aware of and corrected a significantly smaller proportion of everyday action errors than healthy controls. Dementia patients corrected a large proportion of detected errors; however, neither detection nor correction variables were significantly related to overall dementia severity, neuropsychological test performance, or the number of everyday action errors. Although novel, this study had at least two limitations. First, rapid error detection/correction (i.e., instances when patients begin to make an error but detect/correct the action before it is fully executed; hereafter microslips) (Blavier et al., 2005; Oomen \& Postma, 2002) was not examined. Microslips occur frequently in healthy controls (Giovannetti et al., 2007), but their frequency in dementia remains unknown. Second, the time frame between error detection and error correction was not documented. Thus, it is not known whether the rate of error correction also is impaired in dementia patients. The present study addressed these limitations. A group of well-characterized dementia patients diagnosed clinically with Alzheimer's disease (AD), vascular dementia ( $\mathrm{VaD}$ ), or both (i.e., mixed) was recruited. All participants performed a series of everyday tasks and a neuropsychological protocol in the laboratory. Everyday error detection and correction, including microslips and the time of error correction, were coded from videotapes of performance.

The first goal of the study was to characterize error detection and correction processes in the sample. Based on the findings of Giovannetti et al. (2002a), we hypothesized that error detection and correction would be relatively infrequent; however, we expected that our novel examination of microslip error detection might reveal higher rates of error detection/correction than previously reported. Regarding error correction time frames, we reasoned that dementia participants may be more likely to demonstrate delayed corrections than rapid, immediate corrections, because the former are more likely to be driven by environmental cues rather than internal control processes, which may be vulnerable in dementia.

The second goal of this study is to examine the factors related to error detection and correction in dementia patients. Understanding the specific neuropsychological processes related to error monitoring is important in order to understand the nature of detection/correction deficits and inform future rehabilitation techniques. Whereas an earlier study showed no significant relations between error detection/ correction variables and a range of neuropsychological measures (Giovannetti et al., 2002a), a very heterogeneous sample was recruited for that study [e.g., Alzheimer's disease (AD), dementia caused by multiple aetiologies, substance-induced persisting dementia, etc.]. The heterogeneity of the sample may have obscured meaningful relations between error detection/correction and neuropsychological variables. We hypothesized that analyses within a more carefully characterized and relatively more homogeneous dementia sample of $\mathrm{AD}$ and $\mathrm{VaD}$ participants might uncover meaningful relations between error monitoring and neuropsychological variables. Specifically, we predicted that error detection and 
correction would necessitate intact executive functions. However, because error detection and correction have been associated with distinct prefrontal systems (Botvinick et al., 2004; Gehring \& Knight, 2000), we predicted that these constructs may be related to different measures of executive functioning.

\section{METHODS}

\section{Participants}

Participants were selected from a corpus of over 100 participants recruited for prior studies using the Naturalistic Action Test (NAT; Giovannetti et al., 2002b; Giovannetti et al., 2006). All participants had been recruited from one of two similarly structured outpatient memory clinics (Crozer Chester Medical Center, University of Medicine and Dentistry in New Jersey). The clinical evaluation included examination and diagnostic consensus by a neurologist, neuropsychologist, psychiatrist, and a social worker. Inclusion criteria for all earlier studies included evidence of a mild to moderate dementia (Mini-Mental State Examination; MMSE $\geq 10$ and $\leq 26$ ) and diagnosis of probable AD (McKhann et al., 1984), probable/possible ischemic vascular dementia (Chui et al., 1992) (VaD), or both (mixed $\mathrm{AD} / \mathrm{VaD}$ ). For this study, all participants were analyzed as a single group. This decision was based on recent neuropathology studies, which have reported considerable overlap between these diagnostic syndromes (Etiene et al., 1998; Victoroff et al., 1995). Also, these groups did not differ on any monitoring variable (see Appendix).

Participants were excluded from the study if they demonstrated a history of head injury, epilepsy, primary psychiatric illness, cortical stroke, alcohol or illicit substance abuse within the last month, mental retardation, or non-English speaking. All participants signed IRB approved consent forms for their participation in earlier studies.

Healthy controls typically perform at ceiling on the NAT (Schwartz et al., 2003). However, microslips have never been examined with the NAT; therefore data from 6 healthy controls were used as a descriptive source of comparison. Controls were recruited from advertisements in the community. Controls had no subjective complaints of memory problems, no history of dementia or neurological disease/ damage, and obtained MMSE scores >26. All controls signed IRB approved consent forms for their participation.

\section{Procedures and Measures}

As part of their participation in previous studies, all dementia participants completed the NAT following a comprehensive neuropsychological protocol within the same day. Videotapes of NAT performance were analyzed for the detection and correction of errors.

\section{Naturalistic Action Test}

The NAT (Schwartz et al., 2002, 2003) is a standardized measure of naturalistic action that requires completion of three everyday tasks in the laboratory: (1) prepare toast with butter and jelly and prepare instant coffee with cream and sugar; (2) wrap a gift as a present; (3) prepare a lunchbox with a sandwich, snack and a drink, and pack a schoolbag with supplies for school. All objects are available on a U-shaped table, which permits easy view and reach of task items. Because the NAT is designed to assess cognitive rather than motor abilities, physical assistance is permitted when necessary; otherwise, the examiner provides no guidance to the participant. NAT instructions and psychometric properties are described in detail in the manual (Schwartz et al., 2003).

\section{NAT Comprehensive Error Score (CES)}

The NAT manual describes a reliable and extensive coding system for classifying errors (comprehensive error score; CES; (Schwartz et al., 2003; Schwartz et al., 1998). According to the CES, an error is coded when a task step is performed incorrectly (i.e., sequence error, substitution, etc.), when an off-task step is performed (i.e., action addition), and when a step is never executed (i.e., omission). It is important to note that failure to complete large segments of the tasks does not necessarily lead to a reduction in overall error rate; for example, if a participant fails to make toast, he/she is assigned 4 omissions for the following steps: turn toaster on, toast bread, apply butter, apply jelly. The reader should refer to the NAT manual and prior publications for more details on the CES procedures (Giovannetti et al., 2002b; Schwartz et al., 1998; Schwartz et al., 2003).

\section{Microslips}

The CES does not capture errors that are not fully executed (i.e., microslip). However, microslips reflect instances of error detection/correction and are highly relevant to the study of error monitoring; thus they were coded for the present study. Microslips were operationally defined as the initiation and termination of an incorrect action before the error was completed (see also Giovannetti et al., 2007). This included reaching for or picking up an incorrect item or initiating a behavior that was dissonant with the task goal. For example, picking up, but not using, the garden shears instead of the scissors in the gift-wrapping task would be coded as a microslip. For most analyses, microslips were added to CES errors (i.e., Total Errors).

\section{Error Detection and Correction}

For the present study a novel coding system, which was modeled on that of previous studies (Hart et al., 1998), was developed for the analysis of error detection and correction. Two raters (B.M.B. \& L.M.), blind to participant characteristics, independently coded NAT videotapes for microslips, error detection, error correction, and the time frame of error correction. Disagreements between the coders were resolved through discussion and/or re-review of videotapes. Inter- 
Table 1. Error detection and correction coding guide

\begin{tabular}{|c|c|c|}
\hline Monitoring Process & Description & Example \\
\hline \multicolumn{3}{|l|}{ Error Detection } \\
\hline Verbalization & $\begin{array}{l}\text { Individual offers a verbalization indicative } \\
\text { of error recognition or a verbalization } \\
\text { suggestive of possible task mismatch. }\end{array}$ & $\begin{array}{c}\text { "Oops ...", "That's not right . .", "I forgot } \\
\text {...", "I don't know if this is right ..." }\end{array}$ \\
\hline $\begin{array}{l}\text { Failed Attempt } \\
\text { to Correct }\end{array}$ & $\begin{array}{l}\text { Individual demonstrates a failed attempt to } \\
\text { rectify the error. This must include a } \\
\text { physical effort to alter the previously } \\
\text { committed error. }\end{array}$ & $\begin{array}{l}\text { Individual places cookies in a pencil case, } \\
\text { then proceeds to remove the cookies and } \\
\text { place them in a drawer (target }= \\
\text { lunchbox) }\end{array}$ \\
\hline Error Correction & $\begin{array}{l}\text { All corrected errors were considered } \\
\text { detected (see below) }\end{array}$ & \\
\hline \multicolumn{3}{|l|}{ Error Correction } \\
\hline Microslip & $\begin{array}{l}\text { Individual initiates an incorrect action, but } \\
\text { stops himself or herself before the errant } \\
\text { action is completed. }\end{array}$ & $\begin{array}{l}\text { Individuals reaches for, but does not use, } \\
\text { the garden shears instead of the shears. }\end{array}$ \\
\hline Correction & $\begin{array}{l}\text { Individual rectifies an overt error } \\
\text { immediately following the action or after } \\
\text { a delay }\end{array}$ & $\begin{array}{l}\text { Individual prematurely applies a cap to the } \\
\text { thermos, then removes it to add juice. }\end{array}$ \\
\hline
\end{tabular}

rater reliability was assessed for 10 participants selected randomly from the sample. ${ }^{1}$

\section{Error detection}

By definition, all microslips were considered "detected." However, each CES error was further classified as "detected" or "undetected." Error detection was operationally defined as an acknowledgement of mismatch between an individual's executed activity and the prescribed code of behavior for the task. This acknowledgment may present itself in 3 forms: verbalization, failed correction attempt, or an actual correction (see Table 1). To be considered evidence for detection, a verbalization had to indicate recognition of the error or the possibility of task mismatch. (i.e., "I think you said to add sugar and cream to the coffee, but I'm adding only cream."). The second mode of acknowledgement involves physical effort to alter the consequences of the error; however, it does not necessitate that the participant successfully rectify the error. For example, if an individual places cookies in the pencil case, then proceeds to remove them and place them in the drawer (target $=$ lunchbox), the action would be classified as detected, even though the participant failed to accurately correct the error. That is, by removing the cookies from the pencil case, he/she acknowledges the inaccuracy of the action, but fails to properly resolve the problem. Finally, for the purpose of this study, all corrections were considered "detected." The proportion of total errors (i.e., CES + microslips) that was detected was calculated for each participant (Proportion Detected $=$ Total Errors Detected/Total Errors).

${ }^{1}$ Inter-rater reliability for the NAT CES was not assessed because it was available from past records and is not the focus of this study. However, the CES has good reliability, which has been documented in prior publications (Giovannetti et al., 2002a; Schwartz et al., 2003).

\section{Error correction}

Again, all microslips, by definition, were "corrected." Therefore, only CES errors required further coding as either "corrected" versus "uncorrected." A "correction" was coded when an act was accurately "undone." Failed attempts to correct errors were coded as "uncorrected." For example, adding jelly to the bead before toasting, was coded as undetected and uncorrected. This sequence error would have been coded as "detected" and "corrected" if the participant attempted to scrape off the jelly before toasting (i.e., undoing the error). It would have been coded as "detected" and "uncorrected" if the participant made only a verbal comment about the erroneous action ("I know this isn't right").

There is obvious overlap between the detection and correction categories, as we assumed that all corrected errors also were "detected." An error could never be "undetected" but "corrected." Therefore, with respect to correction, we were interested in knowing the proportion of "detected" errors that were subsequently corrected. This was calculated as follows: Proportion Detected-Corrected $=$ Total Errors Corrected/Total Errors Detected.

\section{Error Correction Time Frames}

All corrected errors were further classified according to the behavior(s) that occurred between the error and its correction: microslip, immediate, or delayed. Microslips were the fastest type of correction, as correction occurred before the error was completed. A corrected error was coded as "immediate," when the correction occurred directly after the error, without any intervening actions. Corrected errors were classified as "delayed" when the correction occurred after an additional intermediary or off-task action. For example, when a participant closed the lunchbox without the thermos and 
then packed the pencil case into the schoolbag before correcting the thermos error, then the correction was coded as "delayed." This method does not punish individuals for working slowly —as long as the correction occurs immediately following the error (i.e., the next action), it is labeled "immediate," regardless of the time elapsed. For each participant, the proportion of Total Errors Detected that was coded as "microslip" (Proportion Microslip Correction = Total Microslip/Total Detected), "immediate" (Proportion Immediate Correction $=$ Total Immediate $/$ Total Detected) and "delayed" (Proportion Delayed Correction = Total Delayed/ Total Detected) were calculated.

\section{Neuropsychological Testing}

All participants completed neuropsychological testing as part of their clinical evaluation. From this evaluation, tests were chosen to assess a range of cognitive functions, including overall dementia severity, executive control, language/ naming, and episodic memory. Neuropsychological tests are described in Table 2.

\section{Data analysis}

Summary statistics were performed for all error and detection/ correction variables. Differences among various detection/ corrections variables (e.g., Microslip vs. Immediate vs. Delayed) were assessed using Wilcoxon Signed Ranks Tests, because several variables were non-normal and unable to be transformed. To explore the neuropsychological correlates of detection/correction performance, Spearman Rank Order correlation and regression analysis were conducted. Three participants were missing more than one neuropsychological test score for unknown reasons and were not included in correlation/regression analyses. The BNT was missing for one participant and was replaced using a regression equation derived from the neuropsychological data of participants without missing data. This method also was used to replace the missing PVLT Discriminability score for another participant.

\section{RESULTS}

\section{Characteristics of the Sample}

Dementia participants ( $n=53 ; 29$ AD, $20 \mathrm{VaD}, 4$ mixed) had an average age of 79.5 years $(S D=5.4)$ and 11.8 years of education $(S D=2.3)$. The mean MMSE score was 20.9 ( $S D=3.6$ ), suggesting mild/moderate overall impairment. On average, Controls were 75.6 years old $(S D=9.6)$ and had $13.7(S D=2.7)$ years of education; their mean MMSE score was $28.2(S D=1.3)$

\section{Inter-rater reliability}

The raters demonstrated $98.6 \%$ agreement in coding all errors as detected or corrected (Cohen's kappa $=.96$ ). They consistently agreed on error correction time frames (100\% agreement; Cohen's kappa $=1.0$ ).

\section{NAT errors}

As shown in Table 3, dementia participants made many more CES errors than microslips. Overall, dementia participants made almost six times as many Total Errors as con-

Table 2. Neuropsychological Protocol

\begin{tabular}{|c|c|c|}
\hline Test & Description & References \\
\hline \multicolumn{3}{|l|}{ Dementia Severity } \\
\hline $\begin{array}{l}\text { Mini Mental-State } \\
\text { Examination (MMSE) }\end{array}$ & $\begin{array}{l}30 \text { item measure of dementia severity. Total scores } \\
\text { range from } 0-30 \text {; higher scores indicate better } \\
\text { global cognitive functioning. }\end{array}$ & Folstein et al., 1975 \\
\hline \multicolumn{3}{|l|}{ Executive Functions } \\
\hline $\begin{array}{l}\text { Phonemic word list } \\
\text { generation (FAS) }\end{array}$ & $\begin{array}{l}\text { The dependent variable is the number of words } \\
\text { produced in } 60 \text { seconds beginning with } \mathrm{F} \text {, A, or } \\
\text { S, excluding proper nouns. }\end{array}$ & Spreen \& Strauss, 1998 \\
\hline Clock Drawing Test & $\begin{array}{l}\text { Participants were asked to 1) draw a clock with the } \\
\text { hands set to ten after eleven and 2) copy a } \\
\text { drawing of a clock. Ten possible errors were } \\
\text { scored on each trial. }\end{array}$ & $\begin{array}{l}\text { Cosentino et al., 2004; } \\
\text { Goodglass \& Kaplan, 1983; } \\
\text { Libon et al., 1993, } 1996\end{array}$ \\
\hline \multicolumn{3}{|l|}{ Language } \\
\hline Boston Naming Test & $\begin{array}{l}\text { The dependent variable is the number of pictures } \\
\text { correctly named. }\end{array}$ & Kaplan et al., 1983 \\
\hline \multicolumn{3}{|l|}{ Episodic Memory } \\
\hline $\begin{array}{l}\text { Philadelphia (Repeatable) } \\
\text { Verbal Learning Test- } \\
\text { Discriminability Index } \\
\text { (PVLT-Discriminability) }\end{array}$ & $\begin{array}{l}\text { Participants were asked to remember a } 9 \text { word list, } \\
\text { as on the CVLT. The dependent variable was the } \\
\text { accuracy on the delayed recognition memory } \\
\text { task (Recognition Discriminability). }\end{array}$ & $\begin{array}{l}\text { Garrett et al., 2004; } \\
\text { Price et al., 2004, } 2007\end{array}$ \\
\hline
\end{tabular}


Table 3. Error detection and correction variables

\begin{tabular}{lrr}
\hline \hline & Dementia & Controls \\
& $M(S D)$ & $M(S D)$ \\
\hline NAT Errors & & $2(.89)$ \\
$\quad$ CES Total & $17.34(10.39)$ & $1(1.2)$ \\
$\quad$ Microslips & $2(2.47)$ & $3(1.4)$ \\
$\quad$ Total Errors (i.e., CES + Microslips) & $19.34(11.01)$ & \\
Error Detection & & $73 \%(16.7)$ \\
$\quad$ Proportion Detected (i.e., Total Detected/Total Errors) & $32.7 \%(22.2)$ & $50 \%(30.3)$ \\
$\quad$ Proportion CES Detected (i.e., Total CES Detected/Total CES Errors) & $25.2 \%(19.2)$ & $68.9 \%(19.1)$ \\
$\quad$ Error Correction & & $94.5 \%(13.6)$ \\
$\quad$ Total Proportion Corrected (i.e., Total Corrected/Total Errors) & $24.86 \%(19.02)$ & \\
$\quad$ Proportion Detected-Corrected (i.e., Total Corrected/Total Detected) & $75.8 \%(27.6)$ & $34.7 \%(43.6)$ \\
Error Correction Time Frame & & $55.55 \%(50.2)$ \\
$\quad$ Proportion Microslip Correction (i.e., Total Microslip/Total Detected) & $31.9 \%(30.0)$ & $4.17 \%(10.2)$ \\
$\quad$ Proportion Immediate Correction (i.e., Total Immediate/Total Detected) & $23.8 \%(28.1)$ & $19.9 \%(23.2)$ \\
$\quad$ Proportion Delayed Correction (i.e., Total Delayed/Total Detected) & & \\
\hline \hline
\end{tabular}

trols on the NAT, but produced relatively similar rates of microslip errors. Because of the large discrepancy in error rates between dementia participants and controls, control data were used descriptively, but they were not included in statistical analyses.

\section{Error detection and correction}

On average, dementia participants detected about one-third of their Total Errors (see Table 3). This is somewhat higher than previously reported estimates of error detection that did not consider microslips (i.e., 20.2\% detected reported by Giovannetti et al., 2006). Dementia participants corrected most, but not all, detected Total Errors. Controls showed considerably higher rates of error detection and correction.

\section{Error correction time frame analysis}

Contrary to prediction, dementia participants showed a higher Proportion Microslip Correction than Proportion Delayed Correction $(z=-2.20, p<.05)$. Although Proportion Microslip Correction was greater than Proportion Immediate Correction, the difference was not significant $(z=-1.35, p=.17)$. Furthermore, there was no significant difference between Proportion Immediate Correction and Proportion Delayed Correction. Controls showed a slightly different pattern of corrections, with lower Proportion Delayed Corrections.

\section{Neuropsychological correlates and predictors of error detection/correction}

To evaluate our prediction that error detection and correction are associated with different cognitive processes, Pro- portion Detected and Proportion Detected-Corrected ${ }^{2}$ were included in correlation and regression analyses with neuropsychological variables. Additionally, the neuropsychological correlates of microslips (i.e, Proportion Microslip Correction) were examined separately because this form of rapid error monitoring has never been studied in dementia; thus, we were interested in learning whether this type of monitoring reflected a distinct process. As stated earlier, three participants had more than 1 missing neuropsychological test score and were excluded from these analyses $(n=$ 50). Also, three participants had zero detected errors and therefore had no Proportion Detection-Correction or Proportion Microslip correction score $(n=47)$.

As shown in Table 4, Proportion Detected was moderately and significantly related to one measure of executive functioning: FAS. Proportion Detected-Corrected showed a different pattern of correlations; significant relations were observed with a different executive measure (clock drawing) and naming, and a modest association also was noted with FAS. Proportion Microslip Correction was significantly related to naming, and a modest association was also noted with executive measures.

Regressions were performed to assess the combined effects of neuropsychological predictors on the detection, correction, and microslip variables that were included in the correlation analyses. The best model for Proportion Detected $[F(1,48)=4.5, p=.04]$ accounted for only $9.0 \%$ of the variance and had only FAS as a significant predictor $(\beta=.29, t=2.1, p=.04)$. The regression for Detected-Corrected accounted for slightly more variance

${ }^{2}$ Proportion Detected-Corrected was selected over Total Proportion Corrected for these analyses because it was not significantly nor strongly related to Proportion Detected $(r=-.11, p=.45)$. By contrast, Proportion Corrected and Proportion Detected were significantly and strongly correlated $(r=.90, p<.01)$. 
Table 4. Spearman rank order correlation coefficients among error detection and correction and neuropsychological variables

\begin{tabular}{lccc}
\hline \hline & $\begin{array}{c}\text { Proportion } \\
\text { Detected } \\
(n=50)\end{array}$ & $\begin{array}{c}\text { Proportion } \\
\text { Detected-Corrected } * \\
(n=47)\end{array}$ & $\begin{array}{c}\text { Proportion } \\
\text { Microslips } \\
(n=50)\end{array}$ \\
\hline $\begin{array}{l}\text { Dementia Severity } \\
\quad \text { MMSE }\end{array}$ & .21 & .14 & .20 \\
$\begin{array}{l}\text { Executive Functions } \\
\text { FAS Total Responses }\end{array}$ & $.33^{* *}$ & .27 & .24 \\
$\quad$ Clock Drawing Total Errors & -.26 & $-.28^{* *}$ & $-.29^{* *}$ \\
Language/Memory & & & $.31^{* *}$ \\
$\quad \begin{array}{l}\text { Boston Naming Test } \\
\text { PrVLT Discriminability Index }\end{array}$ & -.01 & .19 & .05 \\
\hline \hline
\end{tabular}

*Proportion Detected-Corrected $=$ Total Corrected $/$ Total Detected

$* * \mathrm{p}<.05$

$[11 \% ; F(1,45)=5.6, p=.02)$ and had only Clock Drawing Test as a significant predictor $(\beta=-.33, t=-2.4$, $p=.02)$. The regression for proportion microslip correction $[F(1,45)=4.6]$ accounted for only $9.2 \%$ of the variance and had only BNT as a significant predictor $(\beta=$ $.30, t=2.1, p=.04)$.

\section{DISCUSSION}

Error-monitoring processes were reliably evaluated in individuals diagnosed with dementia. Consistent with previous findings (Giovannetti et al., 2002a), dementia patients detected a relatively small percentage of their errors, indicating an attenuated ability to recognize and diagnose errors, even in the context of everyday tasks. Despite the low rate of detection, dementia patients corrected a relatively high proportion of the errors that they detected. This has implications for interventions, as dementia patients may benefit specifically from strategies that focus on increasing detection of committed errors.

This study is the first to examine microslips in everyday tasks among dementia participants. Interestingly, the rate of microslips among both dementia patients and controls in this study was lower than expected based on previous reports (Giovannetti et al., 2007). This discrepancy may be explained by methodological differences across studies, as high rates of microslips have been reported among controls when tasks are performed under divided attention and speeded conditions (Giovannetti et al., 2007; Oomen \& Postma, 2002). As expected, the frequency of detection/ correction when including microslips was somewhat higher compared to past studies of only fully committed, overt errors. However, even with microslips, the rate of detection/ correction was still extremely low in dementia relative to controls.

Contrary to prediction, the analysis of correction time frames revealed that when dementia participants corrected their errors, they were more likely to engage in microslip corrections than immediate or delayed corrections. This suggests that detection/correction processes were not substantially slowed in dementia participants and that rapid, automatic detection/correction was relatively preserved. This finding further emphasizes the need to concentrate interventions on improving detection of errors already produced. Strategies designed to increase environmental feedback or offer third-person feedback during task execution might prove to be effective for improving error monitoring in dementia.

Consistent with our prediction, we found that impoverished performance on tests of executive control predicted a diminution in the ability to efficiently detect and correct mistakes. Also as predicted, error detection and error correction variables were related to and predicted by different measures of executive functioning. Detection was associated with the word generation task ("FAS"), whereas correction was related to clock drawing errors. The Clock Drawing Task necessitates constructional abilities, organization, and visual-motor skills, which may be germane to the correction process. Unexpectedly, error correction also correlated with a measure of naming (BNT), but this measure did not significantly predict correction in the regression analysis. Further research is needed to explicate the cognitive processes uniquely associated with detection versus correction, as the executive measures in our study accounted for only a small proportion of variance.

The link between executive control and error detection/ correction is consistent with studies that have shown a relation between informant reported functional decline in older adults and neuropsychological measures of executive control (Cahn-Weiner et al., 2000; Kiosses et al., 2001; Swanberg et al., 2004). However, Giovannetti et al. (2002a) reported that detection/correction abilities were unrelated to everyday action errors on the NAT in dementia. Similarly, in our study, error detection/correction variables were not significantly correlated with NAT Total Errors (i.e., Proportion Detected $r=-.18, p=.18$; Proportion Detected- 
Corrected $r=-.19, p=.18$ ). Thus, future studies should directly examine whether the link between executive functioning and informant reported functional abilities is moderated by error detection/correction abilities. That is, it is conceivable that informants only report or are concerned over everyday action errors that are not corrected by the dementia patient.

Consistent with prior reports (Giovannetti et al., 2002a), dementia severity was not related to any of the detection/ correction variables examined in this study. This suggests that advanced dementia, or global cognitive decline, may not always lead to impaired error detection/correction. Alternatively, error monitoring may rely on specific neuropsychological processes not adequately assessed in a gross measure of functioning. For example, there has been considerable debate regarding the ability of the MMSE to sufficiently detect executive dysfunction, with some studies showing the MMSE is sensitive to executive deficits (Axelrod et al., 1992; Jefferson et al., 2002; van Gorp et al., 1999), and others highlighting the inadequacy of the MMSE in assessing this cognitive domain (Stokholm et al., 2005). Despite this contention, the MMSE clearly does not offer a comprehensive assessment of executive functioning or other processes that might be relevant to monitoring everyday action errors, including task or script knowledge.

The link between microslips and neuropsychological variables also was examined and showed microslips were modestly, but significantly, associated with naming abilities (BNT). This was surprising, as microslip corrections have been described as "pre-attentional," "auto-control" processes that are distinct from the cognitive processes typically assessed with traditional neuropsychological measures (Giovannetti et al., 2007; Oomen \& Postma, 2002). This result could be interpreted as a link between microslips and semantic knowledge. That is, relative preservation of semantic knowledge (i.e., higher BNT score) may be essential to rapidly identify and rectify deviations to the prescribed code of behavior. For example, stopping short of adding sugar, instead of coffee grinds, to a coffee maker is possible only if one possesses and has ready access to the knowledge that sugar is added to brewed coffee, coffee grinds are added to the coffee maker to brew coffee, and so on. Admittedly, this interpretation is speculative; more research is needed to uncover the cognitive mechanisms associated with microslip detection/correction.

The current study faced several limitations. First, we did not have an adequate comparison group, as healthy controls typically perform at or close to ceiling on the NAT. Additionally, control errors are less egregious than those of dementia participants, which may influence the ease with which errors are detected/corrected. Similarly, the relation between error type (e.g., omission, sequence, etc.) and error monitoring in dementia patients was not evaluated in this study, but should be the focus of future research. Second, our sample of $\mathrm{AD}, \mathrm{VaD}$, and mixed participants was only relatively more homogeneous than prior studies (Giovannetti et al., 2002a). Although we found no differences between $\mathrm{AD}$ and $\mathrm{VaD}$ participants on detection/correction variables (see Appendix), future studies should examine error monitoring differences among individuals with different dementia syndromes (e.g., frontal-temporal dementia). Finally, the determination of whether an individual is truly aware of his/her errors remains a challenging, albeit crucial feature of this research. This study relied on verbalizations and failed or successful attempts to correct errors to indicate detection; yet, error awareness may have occurred without overt signs of detection. As a result, error detection variables may have underestimated participants' error awareness.

Despite these limitations, the study also displays several strengths. First, this is the first study to examine correction time frames in everyday error detection/correction in dementia. Second, to our knowledge, this is the first study to examine microslips in dementia; these analyses offered a comprehensive evaluation of monitoring and provided rich detail regarding the efficiency and rapidity of corrective behavior in dementia. Third, the use of videotape afforded a detailed and reliable analysis of monitoring behaviors.

In conclusion, error detection may be more fundamentally impaired in dementia relative to error correction, and individuals with executive control deficits are likely to experience impaired correction/detection in everyday tasks. As a result, interventions for dementia patients should aim to improve error detection. For example, strategies that increase feedback regarding task performance may substantially improve functional abilities in dementia. Finally, future research should investigate the role of semantic knowledge in auto-control, pre-attentional error monitoring.

\section{ACKNOWLEDGMENTS}

This research was conducted in partial fulfillment of the requirements for Brianne Magouirk Bettcher's master's degree. A portion of this paper was presented at the 2007 International Neuropsychological Society conference. We would like to thank Dr. Lauren Alloy and Dr. Nora Newcombe for their helpful comments on an earlier version of this manuscript. This research was supported by funds from the Alzheimer's Association (NIRG-04-1097) and a Research and Study Leave awarded to Tania Giovannetti.

\section{REFERENCES}

Axelrod, B.N., Goldman, R.S., \& Henry, R.R. (1992). Sensitivity of the Mini-Mental State Examination to frontal lobe dysfunction in normal aging. Journal of Clinical Psychology, 48, 68-71.

Biran, I., Giovannetti, T., Buxbaum, L.J., \& Chatterjee, A. (2006). The alien hand syndrome: What makes the alien hand alien? Cognitive Neuropsychology, 23, 563-582.

Blavier, A., Rouy, E., Nyssen, A.S., \& de Keyser, V. (2005). Prospective issues for error detection. Ergonomics, 48, 758-781.

Botvinick, M.M., Cohen, J.D., \& Carter, C.S. (2004). Conflict monitoring and anterior cingulate cortex: An update. Trends in Cognitive Science, 8, 539-546.

Cahn-Weiner, D.A., Malloy, P.F., Boyle, P.A., Marran, M., \& Salloway, S. (2000). Prediction of functional status from neuro- 
psychological tests in community-dwelling elderly individuals. Clinical Neuropsychology, 14, 187-195.

Chui, H.C., Victoroff, J.I., Margolin, D., Jagust, W., Shankle, R., \& Katzman, R. (1992). Criteria for the diagnosis of ischemic vascular dementia proposed by the State of California Alzheimer's Disease Diagnostic and Treatment Centers. Neurology, 42, 473-480.

Cosentino, S., Jefferson, A., Chute, D.L., Kaplan, E., \& Libon, D.J. (2004). Clock drawing errors in dementia: Neuropsychological and neuroanatomical considerations. Cognitive and Behavioral Neurology, 17, 74-84.

Dehaene, S., Posner, M.I., \& Tucker, D.M. (1994). Localization of a neural system for error detection and compensation. Psychological Science, 5, 303-305.

Etiene, D., Kraft, J., \& Ganju, N. (1998). Cerebrovascular Pathology Contributes to the Heterogeneity of Alzheimer's Disease. Journal of Alzheimer's Disease, 1, 119-134.

Folstein, M.F., Folstein, S.E., \& McHugh, P.R. (1975). "Minimental state." A practical method for grading the cognitive state of patients for the clinician. Journal of Psychiatric Research, 12, 189-198.

Garrett, K.L., Price, C., Libon, D.J., Swenson, R., Penney, D.L., Cosentino, S., \& Jefferson, A.L. (2004). Verbal serial list learning among dementia patients with and without white matter changes: Neuropsychological Correlates. Abstract presented at the 32nd annual meeting of the International Neuropsychological Society, Baltimore, MD.

Gehring, W.J. \& Knight, R.T. (2000). Prefrontal-cingulate interactions in action monitoring. Nature Neuroscience, 3, 516-520.

Giovannetti, T., Libon, D.J., Buxbaum, L.J., \& Schwartz, M.F. (2002b). Naturalistic action impairments in dementia. Neuropsychologia, 40, 1220-1232.

Giovannetti, T., Libon, D.J., \& Hart, T. (2002a). Awareness of naturalistic action errors in dementia. Journal of the International Neuropsychological Society, 8, 633-644.

Giovannetti, T., Schmidt, K.S., Gallo, J.L., Sestito, N., \& Libon, D.J. (2006). Everyday action in dementia: Evidence for differential deficits in Alzheimer's disease versus subcortical vascular dementia. Journal of the International Neuropsychological Society, 12, 45-53.

Giovannetti, T., Schwartz, M.F., \& Buxbaum, L.J. (2007). The coffee challenge: A new method for the study of everyday action errors. Journal of Clinical and Experimental Neuropsychology, 29, 690-705.

Goodglass, H. \& Kaplan, E. (1983). The Assessment of Aphasia and Related Disorders (2nd ed.). Philadelphia: Lea and Feibiger.

Hart, T., Giovannetti, T., Montgomery, M.W., \& Schwartz, M.F. (1998). Awareness of errors in naturalistic action after traumatic brain injury. Journal of Head Trauma Rehabilitation, 13, 16-28.

Hartsuiker, R.J. \& Kolk, H.H. (2001). Error monitoring in speech production: A computational test of the perceptual loop theory. Cognitive Psychology, 42, 113-157.

Jefferson, A.L, Cosentino, S.A, Ball, S.K, Bogdanoff, B., Leopold, N., Kaplan, E., \& Libon, D.J. (2002). Errors produced on the Mini-Mental State Exam (MMSE) and neuropsychological test performance among patients with Alzheimer's disease, Ischemic Vascular Dementia, and Parkinson's disease. Journal of Neuropsychiatry \& Clinical Neurosciences, 14, 311-320.

Kaplan, E.F., Goodglass, H., \& Weintraub, S. (1983). The Boston Naming Test (2nd ed.). Philadelphia: Lea \& Febiger.

Kiosses, D.N., Klimstra, S., Murphy, C., \& Alexopoulos, G.S. (2001). Executive dysfunction and disability in elderly patients with major depression. American Journal of Geriatric Psychiatry, 9, 269-274.

Libon, D.J., Malamut, B.L., Swenson, R., Sands, L.P., \& Cloud, B.S. (1996). Further analyses of clock drawings among demented and nondemented older subjects. Archives of Clinical Neuropsychology, 11, 193-205.

Libon, D.J., Swenson, R.A., Barnoski, E.J., \& Sands, L.P. (1993). Clock drawing as an assessment tool for dementia. Archives of Clinical Neuropsychology, 8, 405-415.

Manly, T., Lewis, G.H., Robertson, I.H., Watson, P.C., \& Datta, A.K. (2002). Coffee in the cornflakes: Time-of-day as a modulator of executive response control. Neuropsychologia, 40, $1-6$.

McKhann, G., Drachman, D., Folstein, M., Katzman, R., Price, D., \& Stadlan, E.M. (1984). Clinical diagnosis of Alzheimer's disease: Report of the NINCDS-ADRDA Work Group under the auspices of Department of Health and Human Services Task Force on Alzheimer's Disease. Neurology, 34, 939-944.

Oomen, C.C.E. \& Postma, A. (2002). Limitations in processing resources and speech monitoring. Language \& Cognitive Processes, 17, 163-184.

Postma, A. (2000). Detection of errors during speech production: A review of speech monitoring models. Cognition, 77, 97-132.

Postma, A. \& Kolk, H. (1993). The covert repair hypothesis: Prearticulatory repair processes in normal and stuttered disfluencies. Journal of Speech and Hearing Research, 36, 472-487.

Price, C.C., Garrett, K.D., Jefferson, A.L., Cosentino, S., Bettcher, B.M., Giovannetti, T., Penney, D.L., Swenson, R. \& Libon, D.J. (2007). When does leukaroaisosis (LA) indicate a subcortical dementia? Comparison of LA groups to Huntington's disease on a list-learning paradigm. Abstract submitted to the $3^{\text {rd }}$ biannual meeting of the International Society for Vascular Behaviourial and Cognitive Disorders.

Price, C., Garrett, K.D., Libon, D.J., Swenson, R., Penney, D.L., Jefferson, A.L., \& Cosentino, S. (2004). Verbal serial list learning among dementia patients with and without white matter changes: Factor Solutions. Abstract presented at the 32nd annual meeting of the International Neuropsychological Society, Baltimore, MD.

Rabbit, P. (1978). Detection of errors by skilled typists. Ergonomics, 21, 945-958.

Reason, J. (1990). Human Error. Cambridge: Cambridge University Press.

Schwartz, M.F., Buxbaum, L.J., Ferraro, M., Veramonti, T., \& Segal, M. (2003). The Naturalistic Action Test. Bury St. Edmunds, UK: Thames Valley Test Company.

Schwartz, M.F., Montgomery, M.W., Buxbaum, L.J., Lee, S.S., Carew, T.G., Coslett, H.B., Ferraro, M., Fitzpatrick-DeSalme, E., Hart, T., \& Mayer, N. (1998). Naturalistic action impairment in closed head injury. Neuropsychology, 12, 13-28.

Schwartz, M.F., Segal, M., Veramonti, T., Ferraro, M., \& Buxbaum, L.J. (2002). The Naturalistic Action Test: A standardised assessment for everyday action impairment. Neuropsychological Rehabilitation, 12, 311-339.

Spreen, O. \& Strauss, E. (1991). A compendium of neuropsychological tests: Administration, norms and commentary. New York: Oxford University Press.

Stokholm, J., Vogel, A., Gade, A., \& Waldermar, G. (2005). The executive interview as a screening test for executive dysfunction in patients with mild dementia. Journal of the American Geriatrics Society, 53, 1577-1581 
Swanberg, M.M., Tractenberg, R.E., Mohs, R., Thal, L.J., \& Cummings, J.L. (2004). Executive dysfunction in Alzheimer disease. Archives of Neurology, 61, 556-560.

van Gorp, W.G, Marcotte, T.D., Sulzer, D., Hinkin, C., Mahler, M., \& Cummings, J.L. (1999). Screening for dementia: Comparison of three commonly used instruments. Journal of Clinical and Experimental Neuropsychology, 21, 29-38.
Victoroff, J., Mack, W.J., \& Lyness, S.A. (1995). Multicenter clinicopathological correlation in dementia. American Journal of Psychiatry, 152, 1476-1484.

Zapf, D. \& Reason, J.T. (1994). Introduction: Human errors and error handling. Applied Psychology: An International Review, 43, 427-432.

\section{Appendix: Error Detection and Correction in AD versus VaD}

Participants diagnosed with Alzheimer's disease $(n=29)$ and vascular dementia $(n=20)$ did not significantly differ in age $(z=-.12, p=.90)$, education $(z=-1.21, p=.23)$, or dementia severity $(z=-1.90, p=.06)$. Between-group analyses were performed for all error detection and correction variables using Mann-Whitney $U$ tests. As shown in Table A1, the AD and VaD participants did not differ on any of the error monitoring variables.

Table A1. Error Detection and Correction in Alzheimer's Disease and Vascular Dementia Patients

\begin{tabular}{lccc}
\hline \hline & $\mathrm{AD}$ & $\mathrm{VaD}$ & \\
& $M(S D)$ & $M(S D)$ & Mann-Whitney U \\
\hline $\begin{array}{c}\text { Proportion Detected } \\
\quad \text { Total Detected/Total Errors) }\end{array}$ & $33.74(20.83)$ & $33.55(23.87)$ & $z=-.122, p=.90$ \\
$\begin{array}{c}\text { Proportion Detected-Corrected } \\
\quad \text { Total Corrected/Total Detected) }\end{array}$ & $74.13(29.65)$ & $78.55(21.81)$ & $z=-.163, p=.87$ \\
$\begin{array}{c}\text { Proportion Microslip Correction } \\
\quad \text { (Total Microslip/Total Detection) }\end{array}$ & $32.50(29.81)$ & $36.01(31.41)$ & $z=-.340, p=.73$ \\
$\begin{array}{c}\text { Proportion Immediate Correction } \\
\quad \text { (Total Immediate/Total Detection) }\end{array}$ & $20.00(23.37)$ & $23.31(25.85)$ & $z=-.313, p=.76$ \\
$\begin{array}{c}\text { Proportion Delayed Correction } \\
\quad(\text { Total Delayed/Total Detection) }\end{array}$ & $21.35(22.76)$ & $19.23(25.61)$ & $z=-.642, p=.52$ \\
\hline
\end{tabular}

